\title{
Artificial Neural Network Based Hybrid Algorithmic Structure for Solving Linear Programming Problems
}

\author{
L.R. Arvind Babu and B.Palaniappan
}

\begin{abstract}
Linear Programming Problems are mathematical models used to represent real life situations in the form of linear objective function and constraints various methods are available to solve linear programming problems. When formulating an LP model, systems analysts and researchers often include all possible constraints although some of them may not be binding at the optimal solution. The presence of redundant constraints does not alter the optimum solution(s), but may consume extra computational effort. Redundant constraints identification methods are applied for reducing computational effort in LP problems. But accuracy of the LP problems goes down due to this reduction of loops and constraints. To achieve optimality in accuracy and also in computational effort, we propose an algorithm, called, Hybrid Algorithm, it trains the constraint and parameter before applying the formal methodology.
\end{abstract}

Index Terms-Linear Programming , Redundant Constraints, Load Forecasting , Training Parameters.

\section{INTRODUCTION}

Linear programming (LP) is one of the most important techniques used in modeling and solving practical optimization problems that arise in industry, commerce, and management. Linear Programming Problems are mathematical models used to represent real life situations in the form of linear objective function and constraints various methods are available to solve linear programming problems.

When formulating an LP model, systems analysts and researchers often include all possible constraints although some of them may not be binding at the optimal solution. The presence of redundant constraints does not alter the optimum solution(s), but may consume extra computational effort. Many researchers have proposed algorithms for identifying the redundant constraints in LP models.

The widely used methodology for LP problems is revised simplex method, a univariate search technique. It suffers the drawback of slow convergence with the tendency of variable popping in and out of the basis matrix. The numbers of iterations are more and increase in computational effort and time. This gives wide scope to a search of new algorithm. In the bounded complex algorithm [12] a univariate search technique is adopted, but the entering variables are arranged not only based on the maximum contribution if that variable to the objective function but also on the type of the constraints. Once the priority arrangement of the promising variables is obtained the iterations are performed as in the
Simplex Method by selecting a leaving variable based on bounds of the variables.

Redundancies, if any, in the LP model will waste computational effort. In fact, there is a wide literature on the detection and elimination of redundant constraints in LP models [1-11]. Heuristic approach [13] is using an intercept matrix to identify redundant constraints prior to the start of the solution process. In this heuristic approach, tendency of variables to pop in and pop out of the basis is eradicated after eliminating the redundancies. The eradication of pop-in and pop-out substantially reduces the number of iterations. A significant reduction in the computational effort is achieved for LP problems of different sizes in heuristic approach.

\section{REDUndant Constraints AND COMPLEX Algorithm}

\section{A. Identification of Redundant constraints}

Redundant constraints are the constraints that can be omitted from a system of linear constraints without changing the feasible region.

Consider the following system of $m$ linear inequality constraints and $n$ variables $(m \geq n)$ :

$$
A X \leq b, X \geq 0
$$

where $A \in R m \times n, b \in R m, X \in R n$ and $0 \in R n$.

The feasible region $S$ associated with system (1) is defined as

$$
S=\{X \in R n / A X \leq b, X \geq 0\} .
$$

Redundant constraints in system (1) do not play a role in determining the feasible region $\mathrm{S}$.

To define redundant inequality constraints more formally, we denote for any fixed $k \in(1,2,3, \ldots, m), S_{k}=\{X \in$ $R n / A i X \leq b i, X \geq 0 \forall i \neq k\}$.

The $k$ th constraint $A_{k} X \leq b_{k}(1 \leq k \leq m)$ is a redundant inequality in system (1) if and only if $S_{k}=S$.

\section{B. Complex Algorithm}

Very often a linear programming problem may have some or all variables bounded with lower and upper limits in addition to the given constraints. The lower and upper limits can represent the minimum and maximum demands for certain products to solve this kind of these problems a special kind of technique known as a bounded variable simplex method is available. A new algorithm is presented to solve linear bounded variable problems whose constraints coefficient is non-negative. In this method the variable are 
arranged according to their total contribution to the objective function as was done in solving linear programming problem.

1) If any variable has a lower bound. Then substitute $X^{1}=$ $\mathrm{L}+\mathrm{X}$.

2) Determination of entering vector $P_{j}$

$$
\mathrm{Z}_{\mathrm{j}} \mathrm{C}_{\mathrm{j}}=\left(1 C_{B} B^{-1}\right)\left(\begin{array}{l}
-c_{j} \\
P_{j}
\end{array}\right)
$$

If the objective function is maximization a variable having $\left(\mathrm{Z}_{\mathrm{j}}-\mathrm{C}_{\mathrm{j}}\right)$ value as negative is Promising variable. If the objective function is maximization a variable having $\left(Z_{j}-C_{j}\right)$ value as positive is Promising variable.

3) Determination of leaving vector $\mathrm{P}_{r}$ when the entering vector is $P_{j}$ and the current basis matrix $B_{c}$, the leaving vector must correspond to $\theta_{0}=\min \left[\theta_{1}, \theta_{2}, \mu_{j}\right]$ where

$$
\theta_{1}=\min _{k}\left(\frac{B^{-1} P_{0}}{\alpha_{k j}} ; \alpha_{k j}>0\right)
$$

Where $\alpha_{k j}=B^{-1} P_{j}, K=1,2, \ldots \ldots . ., m$

$$
\theta_{2}=\min _{k}\left(\frac{\mu_{k}-\left(B^{-1} P_{0}\right)}{\alpha_{k j}} ; \alpha_{k j}<0\right)
$$

4) After selecting the leaving variable using the above condition the changes in the current basis solution can be effected as follows

a) If $\theta=\theta_{1}$ which correspond $\mathrm{i}=\mathrm{r}$ than variable $\mathrm{X}_{\mathrm{j}}$ enters the basis while the variable corresponds to $\left(\mathrm{B}^{-1} \mathrm{P}_{0}\right)_{\mathrm{r}}$ leaves the basis. Update $\mathrm{M}^{-1}$ using step 5 .

b) If $\theta=\theta_{2}$ then $\mathrm{X}_{\mathrm{j}}$ enters the basis and the variable $\left(\mathrm{B}^{-1} \mathrm{P}_{0}\right)_{\mathrm{r}}$ leaves the basis. When $\left(\mathrm{B}^{-1} \mathrm{P}_{0}\right)_{\mathrm{r}}$ is non-basic, its value must be zero. To achieve this put $\left(\mathrm{B}^{-1} \mathrm{P}_{0}\right)_{\mathrm{r}}=\mu_{\mathrm{r}}-\left(\mathrm{B}^{-1} \mathrm{P}_{0}\right)_{\mathrm{r}}{ }^{1}$ and $\mathrm{P}_{0}$ becomes $\mathrm{P}_{\mathrm{o}}{ }^{1}=\mathrm{P}_{0}-\mu_{\mathrm{j}} \mathrm{P}_{\mathrm{j}}$ and update $\mathrm{M}^{-1}$ using step 5. If $\theta=\mu_{j}$ then put $\mathrm{X}_{\mathrm{j}} \mu_{j}-X_{j}^{1}$ then entering variable is non-basic at $\mathrm{X}_{\mathrm{j}}=\mu_{j}$, there is no variable leaving the basis. But it creates the following changes $P_{j}{ }^{1}=-P_{j}$ and $P_{o}$ becomes $P_{0^{-}}$ $\mu_{j} P_{j}$ and $Z_{j}-C_{j}=Z_{j}-C_{j}$.

5) Construction of $\mathrm{M}^{-1}$

Obtain the column vector $\eta$ for the leaving $\mathrm{X}_{\mathrm{j}}$ defined by

$$
\eta=\left[\begin{array}{c}
Z_{j} C_{j} \\
B^{-1} P_{j}
\end{array}\right]
$$

6) If the list of ordered variable is exhausted then go to step 7 , else go to step 2 .
7) If any vector enters at its upper bound take the negated column then go step 2, else terminate the process.

\section{HYBRID ALGORITHM}

A most excellent algorithm must be optimal in both computational effort and accuracy. All the above methodologies concentrated only on achieving best

computational effort. Where as accuracy of these systems may goes down due to reducing number of constraints and number of iteration. To achieve optimality in computational effort and also in accuracy, we proposed a hybrid algorithmic structure.

If we reduce number of iterations, the time complexity become optimal, but the accuracy of the system has reduced. To improve the accuracy, we suggested training and learning of parameters and constraints. This training is possible in real world application by applying artificial neural network (ANN).The ANN has applied for various applications to predict forecasting of parameters, constraints and also to obtain optimality in real world parameters. To proceed further, we consider a real world application, for ex. Load forecasting. Load forecasting is essential in the electricity market for the participants to manage the market efficiently and stably. However, the electric power load forecasting problem is not easy to handle due to its nonlinear and random-like behaviors of system loads, weather conditions, and variations of social and economic environments, etc. Many studies have been reported to improve the accuracy of load forecasting using the conventional methods such as regression-based method [14], Kalman filter [15], and knowledge-based expert system [16]. However, these techniques have a possibility to lack the accuracy of prediction with the higher load forecasting errors in some particular time zones, which are, for example, the weekdays of the summer season, weekend, and/or Monday. To overcome this problem, the computational intelligence techniques [16]-[24], which are the fuzzy systems and artificial neural networks[19,22,27], have been investigated in the past decade as an alternative to the conventional methods.

\section{4-hour daily load forecasting is as follows:}

Step 1). If the predicted day is belonging to a summer season, go to step 4). Otherwise, it is the day on spring, fall, or winter seasons.

Step 2). Construct input information using the load data during three days (which are subject to Monday through Friday) before the predicted day.

Step 3). Forecast the maximum load using the exponential smoothing method.

Step 4). In case that the predicted day belongs to a summer season, the temperature sensitivities are computed using the variations of the load and temperature between the predicted day and its one previous day.

Step 5). Forecast the maximum load with the temperature sensitivities calculated in step 4). After taking the above steps, the normalized value of the 24

hourly loads is calculated from the data obtained from the 
load during the previous three weeks of the predicted day. Thereafter, the 24 hourly loads of the day are forecasted from the normalized value.

Our proposed generalized algorithm is as follows:

Step 1). If the predicted day / time are belonging to class-1 environment, go to step 4). Otherwise, it is class-2 environment.

Step 2). Construct input information using the input parameters and constraints using any formal or conventional methods like complex algorithm and or identifying redundant constraints before the predicted day or time.

Step 3). Forecast the appropriate parameters.

Step 4). In case that the predicted day or time belongs to a class- 1 situation, the value of parameters may vary.

Step 5). Forecast the appropriate parameters with the physical sensitivities calculated in step 4). After taking the above steps, the normalized value of parameters is calculated from the data obtained. Thereafter, the parameters for any class of situation are forecasted from the normalized value.

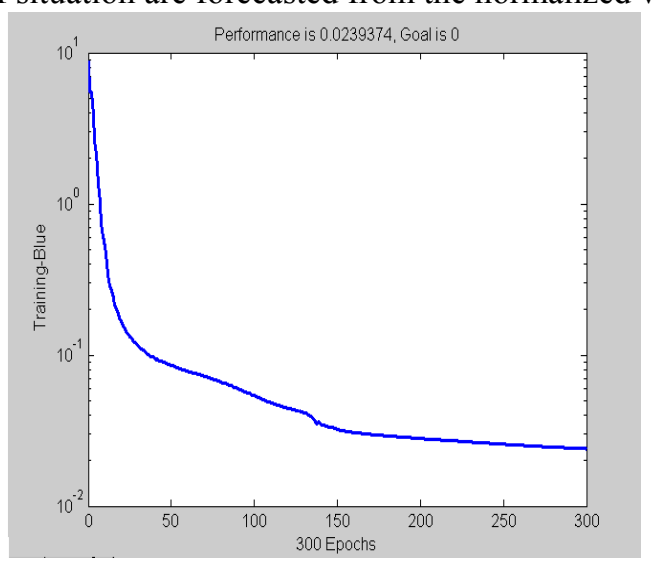

Fig. 2 Result of Back propagation in bounded Variables and Redundant constraints

\section{RESUlt AND CONCLUSION}

We proposed a hybrid algorithmic structure that improves the accuracy of bounded variables in linear programming problem model by suggesting the training and learning of parameters and constraints. This training is possible in real world application by applying artificial neural network. The above figures shows the results of back propagation in bounded variables and redundant constraints, error rate in linear programming problem model. We believe, the proposed algorithm shows increased performance in accuracy.

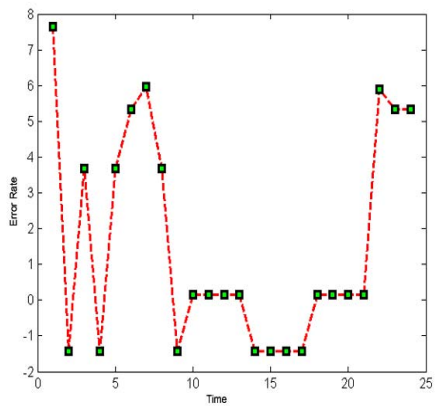

Fig. 3 Error Rate in Linear programming problem model

\section{REFERENCES}

[1] Anderson, E.D. andAndersen K.D., 1995, Presolving in linear programming. Mathematical Programming Series B, 71, 221-245.

[2] Brearley, A.L., Mitra, G. and Williams, H.P., 1975, Analysis of mathematical programming problem prior to applying the simplex algorithm. Mathematical Programming, 8, 54-83.

[3] Gowdzio, J., 1997, Presolve analysis of linear program prior to applying an interior point method. INFORMS:Journal on Computing, 9, 73-91.

[4] Ioslovich, I., 2002, Robust reduction of a class of large scale linear program, SIAM Journal on Optimization, 12, 262-282.

[5] Karwan, M.H., Loffi, V., Telgan, J. and Zionts, S., 1983, Redundancy in Mathematical Programming: A Stateof the Art Survey (Berlin Springer-Verlag).

[6] Kuhn, H.W. and Quandt, R.E., 1962,An experimental study of the simplex method. In: N. Metropolis et al. (Eds) Proceedings of Symposia in Applied Mathematics,Vol. 15, pp. 107-124 (Providence, RI:American Mathematical Society).

[7] Mattheiss, T.H., 1973, An algorithm for determining irrelevant constraints and all vertices in systems of linear inequalities. Operations Research, 21, 247-260.

[8] Meszaros, C. and Suhl, U.H., 2003, Advanced preprocessing techniques for linear and quadratic programming, OR Spectrum, 25, 575-595.

[9] Stojkovic, N.V. and Stanimirovic, P.S., 2001, Two direct methods in linear programming. European Journal of Operational Research, 131, $417-439$.

[10] Telgan, J., 1983, Identifying redundant constraints and implicit equalities in system of linear constraints. Management Science, 29, 1209-1222.

[11] Tomlin, J.A. andWetch, J.S., 1986, Finding duplicate rows in a linear programming model. Operations Research Letters, 5, 7-11.

[12] L.R. Arvind Babu, AM. Sameeullah and B. Palaniappan., 2007, Complex algorithm for bounded variables. Acta Ciencia Indica, vol XXXIII M, No. 2, 633 .

[13] S. Paulraj, C. Chellappan, T. R. Natesan, 2006, A heuristic approach for identification of redundant constraints in linear programming models. International Journal of Computer Mathematics, 83:8, $675-$ 683.

[14] A. D. Papalexopoulos and T. C. Hesterberg, "A regression-based approach to short-term system load forecasting," IEEE Trans. Power Syst., vol. 4, no. 4, pp. 1535-1547, Nov. 1990.

[15] D. J. Trudnowski et al., "Real-time very short-term load prediction for power-system automatic generation control," IEEE Trans. Control Syst. Technol., vol. 9, no. 2, pp. 254-260, Mar. 2001.

[16] S. Rahman and R. Bhatnagar, "An expert system based algorithm for short-term load forecast," IEEE Trans. Power Syst., vol. 3, no. 2, pp. 392-399, May 1988

[17] A. G. Bakirtzis et al., “A neural network short-term load forecasting model for the Greek power system," IEEE Trans. Power Syst., vol. 11 no. 2, pp. 858-863, May 1996.

[18] R. Lamedica et al., "A neural network based technique for short-term forecasting of anomalous load periods," IEEE Trans. Power Syst., vol. 11, no. 4, pp. 1749-1756, Nov. 1996.

[19] K. H. Kim, H. S. Youn, and Y. C. Kang, "Short-term load forecasting for special days in anomalous load conditions using neural networks and fuzzy inference method," IEEE Trans. Power Syst., vol. 15, no. 2, pp. 559-565, May 2000.

[20] H. Mori and A. Yuihara, "Deterministic annealing clustering for ANNbased short-term load forecasting," IEEE Trans. Power Syst., vol. 16, no.3, pp. 545-551, Aug. 2001.

[21] T. Senjyu, H. Takara, and T. Funabashi, "One-hour-ahead load forecasting using neural network," IEEE Trans. Power Syst., vol. 17 no. 1, pp. 113-118, Feb. 2002

[22] K. B. Song, Y. S. Baek, D. H. Hong, and G. S. Jang, "Short-term load forecasting for the holidays using fuzzy linear regression method," IEEE Trans. Power Syst., vol. 20, no. 1, pp. 96-101, Feb. 2005.

[23] J. Nazarko and W. Zalewski, "The fuzzy regression approach to peak load estimation in power distribution systems," IEEE Trans. Power Syst., vol. 14, no. 3, pp. 809-814, Aug. 1999

[24] [24] H. S. Park, K. J. Mun, H. S. Kim, G. H. Hwang, H. S. Lee, and J. H. Park, "Application of neural networks to short-term load forecasting using electrical load pattern,” KIEE Trans., vol. 48A, no. 1, pp. 8-13, 1999.

[25] [25] Y. Yoon et al., "Development of the Integrated System for Power System Operational Planning and Analysis," KEPRI, Tech. Rep. TR.94YJ 15.J1998.89, Dec. 1998. 
[26] [26] H. Tanaka and J. Watada, "Possibilistic linear system and their application to linear regression model," Fuzzy Sets Syst., vol. 27, pp. 275-289, 1988.

[27] [27] D. H. Hong et al., "Fuzzy linear regression analysis for fuzzy inputoutputdata using shape preserving operations," Fuzzy Sets Syst., vol. 122, pp. 513-526, Sep. 2001.

L.R. Arvind Babu was born in Cuddalore 1975, he obtained B.E., in Instrumentation Engineering and M.Sc., Information Technology from
Annamalai University in 1998 and 2001 respectively, he is presently assistant professor in computer science \& engineering ,Annamalai University

B. Palaniappan was born in 1945, he obtained B.E., Electrical Engg., and M.Sc., Electrical Engg., from Annamalai University, he is presently Head,Dept. of Computer Science and Engg., \& Dean, Faculty of Engg., Annamala

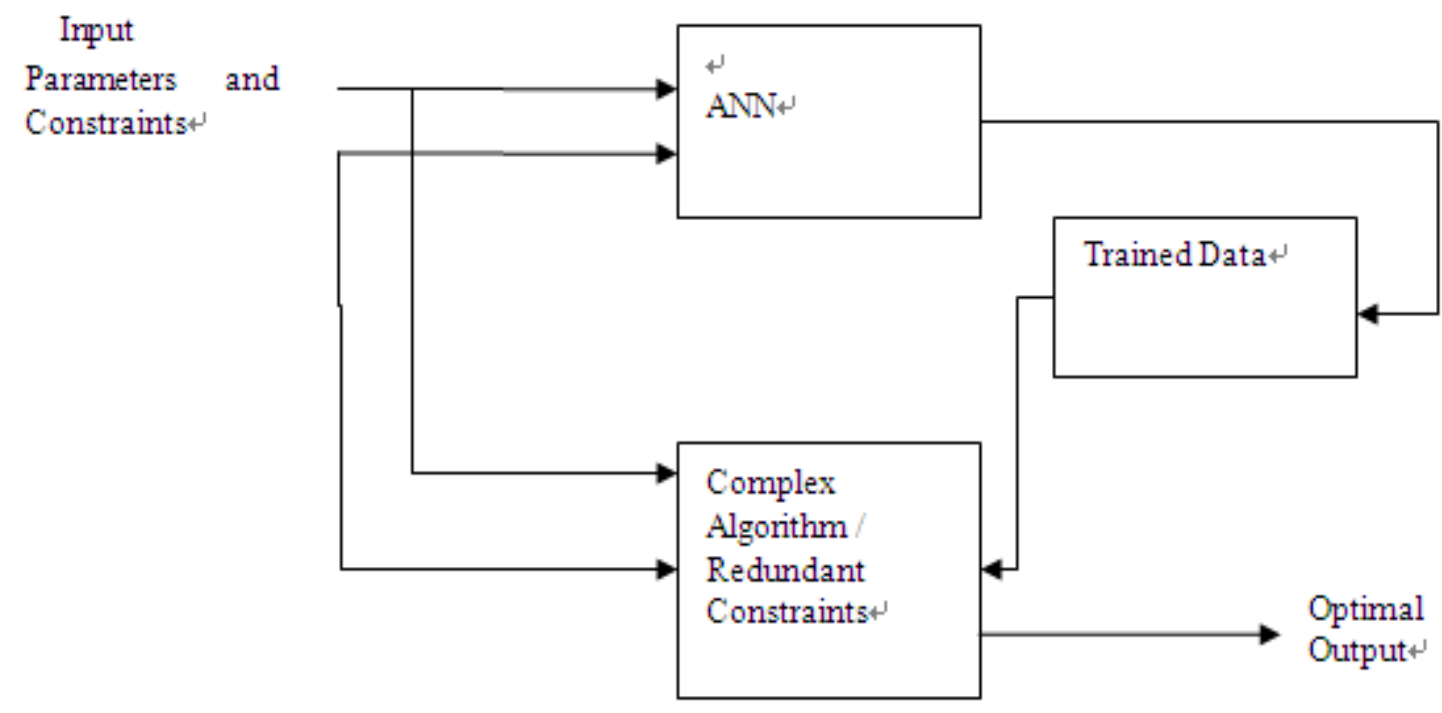

Fig 1. Proposed Hybrid Structure (Where Complex algorithm / redundant constraints is used to achieve optimal in computational effort where as ANN is applied to achieve accuracy in any process model). 